\title{
Fatores de risco e tuberculose: alerta para os profissionais de saúde
}

\author{
Risk factors and tuberculosis: alert to health professionals
}

Milena Nunes Alves de Sousa ${ }^{1,2 *}$, Antônio Mateus Máximo da Silva ${ }^{1}$, Pammela Rikelly França Alves $^{1}$, Patrícia Ferreira Fausto ${ }^{1}$, Thuany Rodrigues Dias ${ }^{1,3}$, Larissa de Araújo Batista Suarez ${ }^{2,4}$, Marriane Brito Macedo ${ }^{1}$, André Luiz Dantas Bezerra ${ }^{1,2}$

\begin{abstract}
RESUMO
\section{RESUMO}

Objetivou-se identificar os fatores de risco para a infeção, o abandono do tratamento e a recidiva da tuberculose. Foi executada, em outubro de 2021, uma revisão sistemática do tipo integrativa, com buscas na Literatura Latino-Americana e do Caribe em Ciências da Saúde e Base de Dados de Enfermagem. Para tanto, foi utilizada a combinação <<tuberculose $A N D$ "fatores de risco">>, selecionando-se 10 artigos após triagem aos pares e aplicação dos critérios de elegibilidade. A maioria dos artigos selecionados foi de estudos transversais, brasileiros e do recorte temporal de 1998-2021. Quanto aos fatores de risco relacionados à infecção destacaram-se: idade, ocupação, tabagistas e alcoolistas. Os de abandono do tratamento foram: sexo masculino, adultos jovens, ambiente socioeconômico, presença de comorbidades e apresentar forma clínica pulmonar. Relacionando-se a recidiva, as irregularidades do paciente foram as mais citadas. Torna-se categórico que os profissionais de saúde possam ser mais bem orientados quanto aos desafios no manejo da tuberculose, em que o conhecimento dos fatores de risco é fundamental para assegurar a prevenção do agravo e a promoção da saúde de grupos vulneráveis.
\end{abstract}

Palavras-chave: Tuberculose; Fatores de Risco; Recidiva; Prevenção de Doenças; Promoção da Saúde.

\begin{abstract}
The objective of this study was to identify the risk factors for infection, treatment abandonment and recurrence of tuberculosis. In October 2021, a systematic review of the integrative type was carried out, with searches in Latin American and Caribbean Literature in Health Sciences and Nursing Databases. For this, the combination <<tuberculosis and "risk factors"〉> was used, selecting 10 articles after peer screening and application of the eligibility criteria. Most of the articles selected were cross-sectional, Brazilian and temporal studies from 1998-2021. As for the risk factors related to the infection, the following were highlighted: age, occupation, smokers and alcoholics. Those of treatment abandonment were: male, young adults, socioeconomic environment, presence of comorbidities and clinical pulmonary form. In relation to recurrence, the patient's irregularities were the most cited. It is categorical that health
\end{abstract}

\footnotetext{
${ }^{1}$ Centro Universitário de Patos (UNIFIP), Patos, Paraíba, Brasil.

*E-mail: milenanunes@fiponline.edu.br

${ }^{2}$ Faculdade São Francisco da Paraíba (FASP), Cajazeiras, Paraíba, Brasil.

${ }^{3}$ Universidade Federal do Rio Grande do Norte, Caicó, Rio Grande do Norte, Brasil.

${ }^{4}$ Universidade Católica do Pernambuco, Recife, Pernambuco, Brasil.
} 
professionals can be better guided about the challenges in the management of tuberculosis, in which knowledge of risk factors is fundamental to ensure the prevention of the disease and the promotion of the health of vulnerable groups.

Keywords: Tuberculosis; Risk factors; Recurrence; Disease Prevention; Health Promotion.

\section{INTRODUÇÃO}

Causada pelo Mycobacterium tuberculosis ou Bacilo de Koch, a Tuberculose (TB) é uma doença infectocontagiosa que possui algumas características peculiares, como seu extenso período de latência entre a infecção inicial até o surgimento de sintomas clínicos da doença (SILVA et al., 2018a; ALENCAR et al., 2019; FERRO, et al., 2019; PASSOS et al., 2019; PEQUENO et al., 2019; SILVA et al., 2019; ARAUJO et al., 2020). Embora tenha predileção pelos pulmões, pode acomete outros órgãos, a exemplo dos rins, e se apresenta como uma resposta granulomatosa juntamente com acentuada lesão tissular (BRASIL, 2019).

É importante ressaltar que, enquanto o paciente estiver liberando bacilos, sem a intervenção do tratamento adequada, a transmissibilidade é plena, levando até 12 meses para manifestação clínica após a infecção inicial. Alerta-se que o paciente com a forma pulmonar é o principal intermédio de infecção, na medida em que o mesmo está eliminando bacilos para o meio externo, podendo contagiar de 10 a 15 pessoas num período equivalente há um ano (BRASIL, 2009).

Segundo Brasil (2014), é interessante destacar a preocupante situação atual da nação brasileira em relação a essa patologia, uma vez que a Organização Mundial de Saúde (OMS) ressalta que o país está entre os 22 países prioritários para gerência e controle da Tuberculose, pois correspondem a $80 \%$ da carga mundial dessa patologia, e, tendo em solo brasileiro, uma média de 5 mil mortos por ano, com distribuição heterogênea entre as regiões.

É relevante avaliar os fatores de risco que estão diretamente ligados à tuberculose dentro do âmbito da saúde pública, quer sejam relacionados à doença, quer sejam ao abandono do tratamento (FRANCO; ZANETTA, 2004; SILVA et al., 2019). Tais fatores oferecem riscos aos profissionais de saúde que lidam diretamente com o manejo do agravo, além de se tratar de pontos relevantes a serem consideradas no seu combate e disseminação. Pesquisa indicou uma frequência de 3,2\% de casos entre 
profissionais do setor de saúde (PEREZ et al., 2020), assim, apresentam risco aumentado de desenvolver a infecção (PRADO et al., 2017).

Bertolozzi et al. (2014) afirmam que a incidência de tuberculose está relacionada a situações como a dificuldade de acesso aos serviços públicos de saúde, tratamento de neoplasias ou uso de fármacos com atividade imunossupressora, comprometimento imunológico, principalmente nos portadores do vírus HIV, desnutrição, idade avançada, entre outros.

Nesta perspectiva, esse estudo busca identificar os fatores de risco para a infeção, o abandono do tratamento e a recidiva da tuberculose proporcionando, assim, a formulação de ações para a prevenção do agravo e promover a saúde das populações vulneráveis.

\section{MATERIAL E MÉTODOS}

Tendo em vista a carência de amplificar e aperfeiçoar a classe das ações de saúde, assim como a da aprendizagem, é importante refletir sobre a seleção e escolha dos estudos. Diferente de anos atrás, em que o embasamento teórico era feito a partir estudos primários, hoje, o alto número de produções científicas diante da mesma temática exige a diligência de revisões sistemáticas (DE-LA-TORRE-UGARTEGUANILO; TAKAHASHI; BERTOLOZZI, 2011).

Assim sendo, adotou-se a revisão sistemática do tipo integrativa, tendo sido construída a partir do Preferred Reporting Items for Systematic Reviews and MetaAnalyses (The PRISMA Statement) (MOHER et al., 2009). No processo de elaboração, é preciso partir da elaboração da pergunta norteadora, tendo sido indagado: "Quais os fatores de risco para a infeção, o abandono do tratamento e a recidiva da tuberculose?".

Em seguida, buscaram-se os Descritores em Ciências da Saúde (DeCS), dando início a pesquisa bibliográfica na Literatura Latino-Americana e do Caribe em Ciências da Saúde (LILACS) e Base de Dados de Enfermagem (BDENF- Enfermagem). Para tanto, foi utilizada a combinação <<tuberculose AND "fatores de risco">>, identificando-se um total de 437 publicações (Figura 1).

Após pesquisa, em que a coleta de dados efetivou-se em outubro de 2021, foi feita a seleção dos critérios de escolha, considerando-se somente documentos na íntegra. Assim, foram pré-selecionadas 300 publicações. Por meio da leitura aos pares dos 
títulos e resumos, excluíram-se os documentos em que não eram artigos, aqueles que os DeCS não estavam no título, que não respondiam a questão de pesquisa e os que se repetiam nas bases de dados. A amostragem final constitui-se por 10 produções científicas na modalidade artigo.

Figura 1 - Fluxograma conforme Recomendação PRISMA referente à seleção das publicações

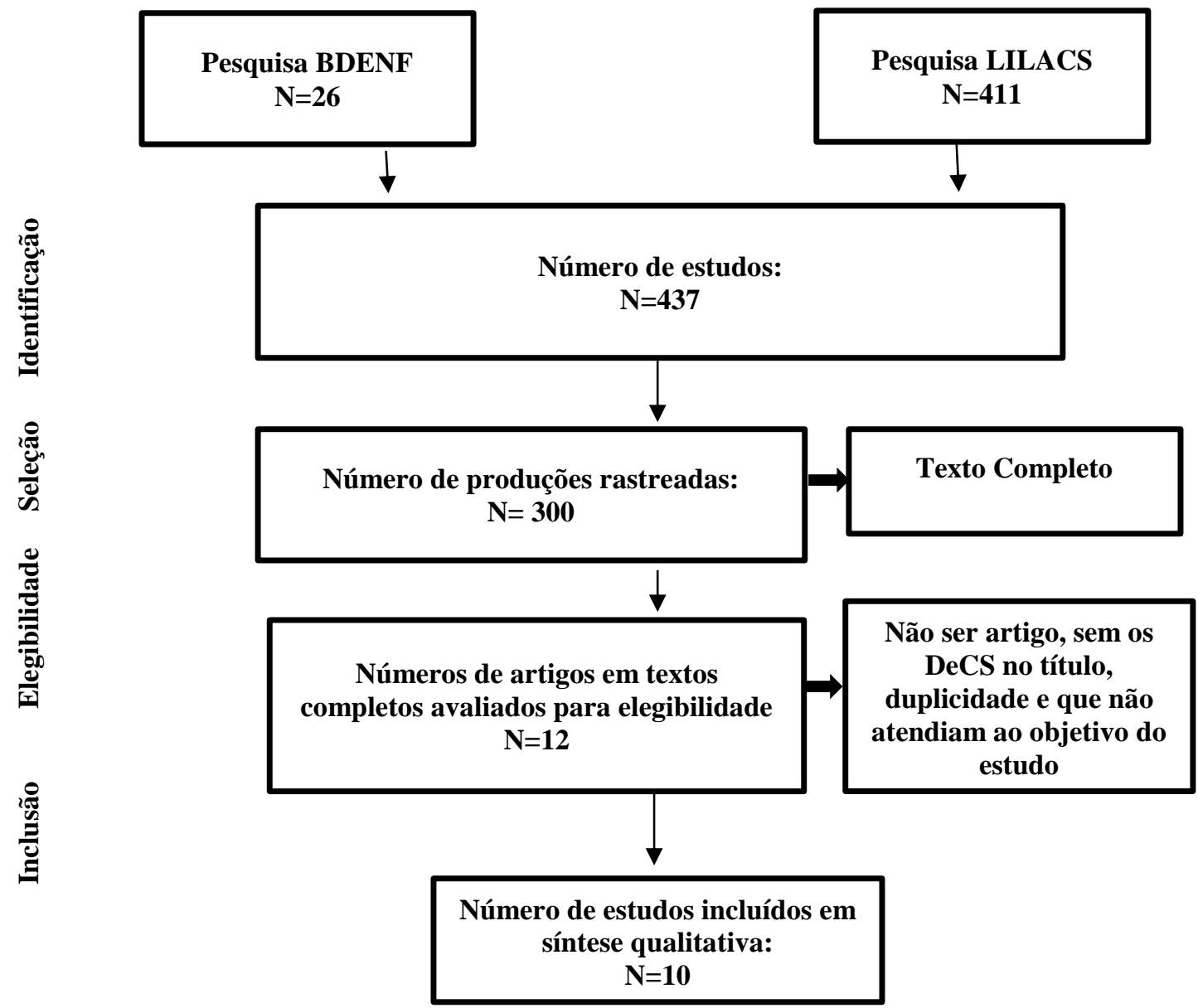

Fonte: Pesquisa em bae de dados, 2021.

Em relação à elucidação dos resultados, foram construídos quadros por meio do Microsoft Word, e selecionadas as variáveis: título, autor/ano, local de publicação, tipo de estudo. Também se extraíram os principais resultados das publicações escolhidas, categorizando-os em: 1) fatores de risco associados à infecção; 2) fatores de risco associados ao abandono do tratamento; e 3) fatores de risco associados à recidiva da infecção. Finalizou-se a RIL com a análise e interpretação dos dados obtidos e a apresentação dos resultados/síntese. 


\section{RESULTADOS}

A maioria dos estudos estava disponível na LILACS (90\%; $n=9)$, publicados em português $(50 \%$; $n=5)$, de pesquisas transversais $(40 \%$; $n=4)$, realizadas no Brasil $(70 \%$; $\mathrm{n}=7$ ) e no recorte temporal de 1998 a 2021.

Quadro 1 - Descrição de títulos, autores/ano, base de dados, tipo de estudo e países

\begin{tabular}{|c|c|c|c|c|}
\hline Autor/ano & Título & $\begin{array}{c}\text { Base de } \\
\text { dados }\end{array}$ & Tipo de Estudo & País \\
\hline $\begin{array}{l}\text { Ferreira } \text { et } \\
\text { al. }(2021)\end{array}$ & $\begin{array}{l}\text { Fatores de risco para o abandono do } \\
\text { tratamento da tuberculose em um } \\
\text { município prioritário amazônico }\end{array}$ & $\begin{array}{c}\text { BDENF - } \\
\text { Enfermagem }\end{array}$ & $\begin{array}{l}\text { Estudo descritivo, } \\
\text { transversal e } \\
\text { quantitativo }\end{array}$ & Brasil \\
\hline $\begin{array}{l}\text { Pérez et al. } \\
\text { (2020) }\end{array}$ & $\begin{array}{l}\text { Factores de riesgo en población no } \\
\text { VIH con tuberculosis en Uruguay }\end{array}$ & LILACS & $\begin{array}{c}\text { Estudo } \\
\text { documental }\end{array}$ & Uruguai \\
\hline $\begin{array}{c}\text { Vaz e } \\
\text { Reifegerste } \\
(2019)\end{array}$ & $\begin{array}{c}\text { Fatores de risco ao abandono do } \\
\text { tratamento da tuberculose em Santa } \\
\text { Catarina }\end{array}$ & LILACS & $\begin{array}{l}\text { Estudo transversal } \\
\text { analítico }\end{array}$ & Brasil \\
\hline $\begin{array}{l}\text { Silva et al. } \\
\quad(2018 b)\end{array}$ & $\begin{array}{l}\text { Risk factors for tuberculosis: diabetes, } \\
\text { smoking, alcohol use, and the use of } \\
\text { other drugs }\end{array}$ & LILACS & Revisão & Vários \\
\hline $\begin{array}{l}\text { Prado et al. } \\
\quad(2017)\end{array}$ & $\begin{array}{c}\text { Prevalence and risk factors for latent } \\
\text { tuberculosis infection among primary } \\
\text { health care workers in Brazil }\end{array}$ & LILACS & Estudo transversal & Brasil \\
\hline $\begin{array}{l}\text { Zenteno- } \\
\text { Cuevas et al. } \\
(2011) \\
\end{array}$ & $\begin{array}{c}\text { Co-infection and risk factors of } \\
\text { tuberculosis in a Mexican HIV+ } \\
\text { population }\end{array}$ & LILACS & Estudo transversal & México \\
\hline $\begin{array}{l}\text { Lannoy et } \\
\text { al. }(2008)\end{array}$ & $\begin{array}{c}\text { Tuberculosis incidence and risk factors } \\
\text { among patients living with HIV/AIDS } \\
\text { in public health service institutions in } \\
\text { Brasilia, Federal District }\end{array}$ & LILACS & $\begin{array}{c}\text { Coorte } \\
\text { retrospectiva }\end{array}$ & Brasil \\
\hline $\begin{array}{c}\text { Picon et al. } \\
(2007)\end{array}$ & $\begin{array}{c}\text { Fatores de risco para a recidiva da } \\
\text { tuberculose }\end{array}$ & LILACS & $\begin{array}{c}\text { Coorte } \\
\text { retrospectiva }\end{array}$ & Brasil \\
\hline $\begin{array}{c}\text { Oliveira e } \\
\text { Moreira- } \\
\text { Filho }(2000) \\
\end{array}$ & $\begin{array}{l}\text { Recidivas em tuberculose e seus fatores } \\
\text { de risco }\end{array}$ & LILACS & $\begin{array}{l}\text { Estudo de caso- } \\
\text { controle }\end{array}$ & Brasil \\
\hline $\begin{array}{l}\text { Menezes et } \\
\text { al. (1998) }\end{array}$ & $\begin{array}{c}\text { Incidência e fatores de risco para } \\
\text { tuberculose em Pelotas, uma cidade do } \\
\text { Sul do Brasil }\end{array}$ & LILACS & $\begin{array}{l}\text { Estudo de caso- } \\
\text { controle }\end{array}$ & Brasil \\
\hline
\end{tabular}

Fonte: Pesquisa em bae de dados, 2021.

Em relação ao quadro 2, notou-se que os fatores de risco estavam relacionados à infecção, ao abandono da terapêutica e com a reinfecção. Entretanto, os estudos destaram-se, em sua maioria, os fatores associados ao abandono do tratamento e a infecção, com $40 \%(n=4)$ das abordagens, cada.

Ao considerar os fatores de risco associados com a infecção (40\%; n=4), destacaram-se: idade, ocupação, tabagistas e alcoolistas. Quanto ao abandono (40\%; $\mathrm{n}=4$ ), merece menção: sexo masculino, adultos jovens, ambiente socioeconômico, 
presença de comorbidades e apresentar forma clínica pulmonar. Relacionando-se a recidiva (20\%; $\mathrm{n}=2)$, as irregularidades do paciente foram as mencionadas pelos autores.

Quadro 2 - Principais fatores de risco relatados pelos autores

\begin{tabular}{|c|c|}
\hline Autor/ano & Fatores de Risco \\
\hline \multicolumn{2}{|r|}{ Fatores de risco associados à infecção } \\
\hline $\begin{array}{l}\text { Prado et al. } \\
(2017)\end{array}$ & $\begin{array}{l}\text { - Ser profissional de saúde, especialmente enfermeiros e técnicos de } \\
\text { enfermagem } \\
\text { - Idade }>50 \text { anos } \\
\text { - Ausência de cicatriz de BCG } \\
\text { - Ex-tabagista } \\
\text { - Uso irregular de máscaras N95 }\end{array}$ \\
\hline $\begin{array}{l}\text { Zenteno- } \\
\text { Cuevas et al. } \\
\text { (2011) }\end{array}$ & $\begin{array}{l}\text { - Sexo masculino } \\
\text { - } \quad \text { Adultos jovens (média de idade de } 35 \text { anos) } \\
\text { - } \quad \text { Solteiros } \\
\text { - } \quad \text { Baixação formal básica } \\
\text { - } \quad \text { Tabagista e Alcoolista } \\
\text { - Desnutridos }\end{array}$ \\
\hline $\begin{array}{l}\text { Lannoy et al. } \\
(2008)\end{array}$ & $\begin{array}{l}\text { - HIV positivo, com a contagem basal de linfócitos T CD4+<200 células/ } / \mu 1 \\
\text { - } \quad \text { Não uso de terapia anti-retroviral. }\end{array}$ \\
\hline $\begin{array}{l}\text { Menezes et al. } \\
(1998)\end{array}$ & $\begin{array}{ll}\text { - } & \text { Pessoas de cor não branca } \\
\text { - } & \text { Aglomeração } \\
\text { - } & \text { Contato com tuberculose } \\
\text { - } & \text { Alcoolismo } \\
\text { - } & \text { Portadores de doenças associadas à tuberculose } \\
\text { - } & \text { Os trabalhadores de pedreiras } \\
\end{array}$ \\
\hline \multicolumn{2}{|r|}{ Fatores de risco associados ao abandono do tratamento } \\
\hline $\begin{array}{l}\text { Ferreira } \text { et al. } \\
(2021)\end{array}$ & $\begin{array}{ll} & \text { Sexo masculino } \\
\text { - } & \text { Adultos jovens (média de idade de } 34 \text { anos) } \\
\text { - } & \text { Cor/raça parda } \\
\text { - } & \text { Baixa escolaridade } \\
\text { - } & \text { Sesempregados } \\
\text { - } & \text { Apresentar forma clínica pulmonar } \\
\text { - } & \text { Raio-x suspeito } \\
\text { - } & \text { Babaciloscopia de escarro positiva } \\
\text { - } & \text { Exames de controle mensal não realizados } \\
\text { - } & \text { Aproximadamente quatro meses de tratamento } \\
& \text { Sem Tratamento Diretamente Observado (TDO) } \\
\end{array}$ \\
\hline $\begin{array}{l}\text { Pérez et al. } \\
(2020)\end{array}$ & $\begin{array}{l}\text { - Ambiente socioeconômico - população de rua, desempregados, usuários de } \\
\text { álcool e outras drogas, desnutrição, contribuindo para a perda de seguimento } \\
\text { do tratamento } \\
\text { - Apresentar forma clínica pulmonar } \\
\text { - Faixa etária (18 a } 35 \text { anos) - adulta jovem } \\
\text { - Presença de comorbidades }\end{array}$ \\
\hline $\begin{array}{ll}\text { Vaz } & \text { e } \\
\text { Reifegerste } & \\
(2019) & \end{array}$ & $\begin{array}{l}\text { - } \quad \text { Idade de } 20 \text { a } 50 \text { anos; } \\
\text { - Não caucasianos } \\
\text { - } \quad \text { Baixa escolaridade } \\
\text { - Reingresso por abandono prévio } \\
\text { - Infecção pelo vírus da imunodeficiência humana (HIV)/Síndrome da } \\
\text { - Imunodeficiência Humana Adquirida (AIDS/SIDA) } \\
\text { - Etilismo }\end{array}$ \\
\hline
\end{tabular}




\begin{tabular}{|c|c|}
\hline $\begin{array}{l}\text { Silva et al. } \\
(2018 b)\end{array}$ & $\begin{array}{ll} & \text { Comorbidades (diabetes) } \\
\text { - } & \text { Tabagismo } \\
\text { - } & \text { Uso de álcool e outras drogas } \\
\end{array}$ \\
\hline \multicolumn{2}{|r|}{ Fatores de risco associados à recidiva da infecção } \\
\hline $\begin{array}{l}\text { Picon et al. } \\
(2007)\end{array}$ & $\begin{array}{l}\text { - } \quad \text { Pacientes HIV-positivos } \\
\text { Pacientes que não aderiram ao tratamento autoadministrado (esquema- } \\
\text { RHZ) }\end{array}$ \\
\hline $\begin{array}{l}\text { Oliveira e } \\
\text { Moreira-Filho } \\
(2000)\end{array}$ & $\begin{array}{l}\text { - Irregularidades do paciente (faltar consulta médica deixando de coletar a } \\
\text { medicação, não ingestão das drogas, doses equivocadas) } \\
\text { - } \text { Idade } \\
\text { - } \text { Estresse gerado por eventos de vida } \\
\text { - Efeitos adversos do uso de drogas antituberculose } \\
\text { - Problemas na organização dos serviços de saúde que implicaram no } \\
\text { fornecimento de dose ou quantidade insuficiente de medicamentos. }\end{array}$ \\
\hline
\end{tabular}

Fonte: Pesquisa em bae de dados, 2021.

\section{DISCUSSÃO}

Os achados desta revisão contemplaram os fatores de risco associados à infecção (MENEZES et al., 1998; LANNOY et al., 2008; ZENTENO-CUEVAS et al., 2011; PRADO et al., 2017), aqueles relacioandos ao abandono do tratamento (SILVA et al., 2018b; VAZ; REIFEGERSTE, 2019; PÉREZ et al., 2020; FERREIRA et al., 2021) e à recidiva da infecção (OLIVEIRA; MOREIRA-FILHO, 2000; PICON et al., 2007).

Entre tantos determinantes relacionados à infeção da tuberculose, nesta revisão, destacaram-se a idade, a ocupação e o hábito tabagista e alcoolista. Muitos autores compartilharam destes achados e acrescentaram outros que merecem ser mencionados, como ser de população carcerária, a co-infecção HIV/TB, as condições/agravos preexistentes e a falta de equipamentos de proteção individual (COSTA et al., 2011; FUKUSHIMA et al., 2011; LAKSHMI et al., 2012; LIMMAHAKHUN et al., 2012; ESTEVAN et al., 2013; ÖNGEN et al., 2013; CHUCHOTTAWORN et al., 2015; SAUNDERS et al., 2017). A associação entre TB-HIV demanda significativa atenção para a realização da quimioprevenção (TEMOTEO et al., 2015).

Pode-se asseverar que a maioria dos fatores de risco está diretamente relacionada com as características individuais/comportamentais e socioeconômicas (COSTA et al., 2011; LAKSHMI et al., 2012; ÖNGEN et al. , 2013; LACERDA et al., 2014; CHUCHOTTAWORN et al., 2015; SAUNDERS et al., 2017). Dentre os aspectos que caracterizam a vulnerabilidade ao adoecimento por TB, revisão sistemática destacou baixos níveis de renda e de escolaridade, idade, condições de moradia, comorbidades, ser indígena, aspectos migratórios, variáveis relacionadas ao trabalho na área da saúde, 
baixa literácia e acesso à saúde (LACERDA et al., 2014). Pode-se somar, ainda, a alta prevalência entre a população carcerária (TAVARES; SOUSA, 2014; SILVA et al., 2016).

A vulnerabilidade social também é um fator determinante do retardo a busca por atendimento médico, sendo a falta de ajuda familiar ou de uma rede de apoio ao cuidado, condições que dificultam o diagnóstico precoce (YEN et al., 2015; LACERDA et al., 2014). Além disso, conforme os autores, o baixo percentual de suspeição e de solicitação de baciloscopia e de outros exames para o diagnóstico da TB também contribuem para a detecção tardia da TB pela APS.

Yen et al. (2015) afirmam que existe uma forte relação entre as precárias condições socioeconômicas (desemprego, baixa escolaridade, baixos salários) e o retardo na busca por serviços de saúde.

Quanto à recidiva da infecção, os autores abordam como fatores de risco as irregularidades do paciente (faltar a consulta médica e coletar a medicação, não ingestão das drogas, doses equivocadas), idade, estresse gerado por eventos de vida, efeitos adversos do uso de drogas antituberculose, além de problemas na oferta e organização dos serviços de saúde (OLIVEIRA; MOREIRA-FILHO, 2000). Soma-se o fato de ser HIV-positivos e de não aderiram ao tratamento autoadministrado (esquema-RHZ) (PICON et al., 2007).

O achado de recidiva é importante, pois se pode inferir que a história patológica pregressa de TB é um dos fatores que predispõe a TB multirresistente (TB-MDR), especialmente nos pacientes com dois ou mais episódios anteriores de TB pulmonar (CHUCHOTTAWORN et al., 2015). Ademais, observou-se uma taxa de baciloscopia de escarro significativamente mais alta nos pacientes com TB-MDR do que aqueles com TB sensível a medicamentos, facilitando assim a disseminação da doença e contaminação dos profissionais (CHUCHOTTAWORN et al., 2015).

Quanto aos problemas relacionados à organização dos serviços de saúde é um dado muito crítico e preocupante, pois o acesso à saúde deve ser assegurando, bem como a integralidade do cuidado. E parece ser uma realidade, pois Melo et al. (2021) identificaram, em estudo, que problemas estruturais e logísticos - falta de materiais ou equipamentos, limitação de locais para a realização dos exames, inexistência de uma conexão entre os serviços envolvidos, entre outros - são comuns. 
Por conseguinte, eesquisa com o objetivo de avaliar o desempenho dos serviços de Atenção Primária a Saúde (APS) em Belo Horizonte em relação às ações de controle da TB constatou que o desempenho satisfatório dos serviços de APS no município foi determinante para resultados positivos na variável abandono do tratamento e grau de risco clínico (RABELO et al., 2021). Destarte, é fundamental superar as fragilidades dos serviços de saúde.

No que tange aos fatores de risco relacionados ao abandono do tratamento (SILVA et al., 2018b; VAZ; REIFEGERSTE, 2019; PÉREZ et al., 2020; FERREIRA et al., 2021), reforça-se que a idade (média 34-35 anos), desempregados, baixa escolaridade, comórbidos (diabetes, especialmente), apresentar forma clínica pulmonar, hábitos de vida (tabagismo, etilismo e consumo de outras drogas), indivíduos com desnutrição, exames de controle mensal não realizados, dente outros.

Estudos associam o diabetes mellitus à tuberculose, com taxa de prevalência que variou entre 6,5\% (LOPES et al., 2020), 7,2\% (ABREU et al., 2020) e 3,23\% a 19,51\% dos casos (SOUSA et al., 2021).

Ademais, Oliveira e Moreira-Filho (2000) reforçaram que ter acesso à informação sobre o tratamento e sua duração constituiu-se em um fator protetivo. Logo, esse reconhecimento e dos demais fatores outra citados deveria implicar em um acompanhamento mais intensivo e maior supervisão para evitar a reativação da tuberculose. Temoteo et al. (2015) e Ferreira et al. (2021) concordam com este posicionamento, ao enfatizarem a relevância do Tratamento Diretamente Observado (TDO).

Por fim, o peso do abandono da terapêutica sobre a epidemiologia da TB demanda ações multidisciplinares (VAZ; REIFEGERSTE, 2019). Não menos importante, fica evidente a necessidade de estratégias para subsidiar o controle da doença, promover saúde e buscar resolutividade para os elementos de vulnerabilidade controláveis (LACERDA et al., 2014; FERREIRA et al., 2021).

\section{CONCLUSÃO}

Os determinantes de risco no manejo da tuberculose estão relacionados à assimilação de oscilantes biológicas, assistenciais e socioeconômicas, desse modo, é imprescindível à sensibilização e mobilização de numerosas partes para construção de 
interposições competentes para diminuir o risco de contágio e o manejar com indivíduos infectados por tuberculose.

É necessário que a rede de atenção ao portador de tuberculose seja organizada, permitindo um cuidado integral e mantendo o acompanhamento da terapêutica. Além disso, o estabelecimento de atividades de vigilância configura-se como fundamental na redução dos fatores de risco, bem como da contaminação e disseminação do agravo, além de permitir o monitoramento do tratamento e identificação precoce da falência terapêutica.

Por fim, os profissionais de saúde precisam ser mais bem orientados quanto aos desafios no manejo da tuberculose, em que o conhecimento dos fatores de risco são fundamentais para assegurar a prevenção do agravo e a promoção da saúde de grupos vulneráveis.

\section{REFERÊNCIAS}

ALENCAR, I. F. P. S. et al. Estratégias preventivas da tuberculose na atenção primária à saúde. Revista Eletrônica Acervo em Saúde, v.11, p.e1297, 2019.

ARAUJO, T. M. et al. Abordagem sobre a tuberculose: análise bibliométrica de periódico de pneumologia. Brazilian Archives of Health and Environment, v.1, n. 1, p.34 - 42, 2020.

BERTOLOZZI, M. R. et al. O controle da tuberculose: um desafio para a saúde pública. Revista Medicina, São Paulo, v.93, n.2, p. 83-89, abr-jun, 2014.

BRASIL. Ministério da Saúde. Secretaria de Vigilância em Saúde. Departamento de Vigilância Epidemiológica. 7. ed. Brasília, DF, 2009.

BRASIL. Ministério da Saúde. Secretaria de Vigilância em Saúde. Departamento de Vigilância das Doenças Transmissíveis. Manual de Recomendações para o Controle da Tuberculose no Brasil. Brasília: Ministério da Saúde, 2019.

CHUCHOTTAWORN, C. et al. Risk factors for multidrug-resistant tuberculosis among patients with pulmonary tuberculosis at the central chest institute of Thailand. PLoS One, v. 10, n. 10, p. e0139986, 2015.

COSTA, J. C. T. et al. Tuberculose ativa entre profissionais de saúde em Portugal. Jornal Brasileiro de Pneumologia, v. 37, p. 636-645, 2011.

DE-LA-TORRE-UGARTE-GUANILO, M. C.; TAKAHASHI, R. F.; BERTOLOZZI, M. R. Revisão sistemática: noções gerais. Revista da Escola de Enfermagem da USP, v. 45, p. 1260-1266, 2011.

ESTEVAN A,O. et al. Active and latent tuberculosis in prisoners in the Central-West Region of Brazil. Rev. Soc. Bras. Med. Trop., v.46, n.4, p.515-518, 2013. 
FERREIRA, M. R. L. et al. Fatores de risco para o abandono do tratamento da tuberculose em um município prioritário amazônico. Rev. Pesqui. (Univ. Fed. Estado Rio J., Online), v. 13, p. 185-191, 2021.

FERRO, A. O. et al. Fitoterapia no Tratamento da Tuberculose. Revista Coopex, v.10, p.1 - 20, 2019.

FRANCO, C.; ZANETTA, D. M. T. Tuberculose em profissionais de saúde: medidas institucionais de prevenção e controle. Arq ciênc saúde, v. 11, n. 4, p. 244-52, 2004.

FUKUSHIMA, Y. et al. Patients in whom active tuberculosis was diagnosed after admission to a Japanese university hospital from 2005 through 2007. Journal of Infection and Chemotherapy, v. 17, n. 5, p. 652-657, 2011.

LACERDA, S. N. B. et al. Individual and social vulnerabilities upon acquiring tuberculosis: a literature systematic review. International Archives of Medicine, v.7, p.35 - 42, 2014.

LAKSHMI, P. V. M. et al. Biomass fuel and risk of tuberculosis: a case-control study from Northern India. J Epidemiol Community Health, v. 66, n. 5, p. 457-461, 2012.

LANNOY, L. H. et al. Tuberculosis incidence and risk factors among patients living with HIV/AIDS in public health service institutions in Brasilia, Federal District. Revista da Sociedade Brasileira de Medicina Tropical, v. 41, p. 549-555, 2008.

LIMMAHAKHUN, S. et al. Treatment outcomes of patients co-infected with tuberculosis and HIV at Chiang Mai University Hospital, Thailand. International journal of STD \& AIDS, v. 23, n. 6, p. 414-418, 2012.

MENEZES, A. et al. Incidência e fatores de risco para tuberculose em Pelotas, uma cidade do Sul do Brasil. Revista Brasileira de Epidemiologia, v. 1, p. 50-60, 1998.

MOHER, D. et al. Preferred reporting items for systematic reviews and meta-analyses: the PRISMA statement. Medicina PLoS, v. 6, n. 7, p. e1000097, 2009.

OLIVEIRA, H. B.; MOREIRA FILHO, D. C. Recidivas em tuberculose e seus fatores de risco. Revista Panamericana de Salud Pública, v. 7, p. 232-241, 2000.

ÖNGEN, G. et al. Pulmonary tuberculosis incidence in Turkish prisons: importance of screening and case finding strategies. Tuberk Toraks, v. 61, n. 1, p. 21-7, 2013.

PASSOS, A. C. et al. Implicações da tuberculose na atenção primária à saúde no Brasil. Revista Coopex, v.10, p.1 - 11, 2019.

PEQUENO, S. F. et al. Vitamina D como agente coadjuvante no tratamento da tuberculose pulmonar. Revista Brasileira de Educação e Saúde. , v.9, n. 4, p.171 - 176, 2019.

PÉREZ, M. et al. Factores de riesgo en población no VIH con tuberculosis en Uruguay. Revista Uruguaya de Medicina Interna, v. 5, n. 1, p. 6-18, 2020.

PICON, P. D. et al. Fatores de risco para a recidiva da tuberculose. Jornal Brasileiro de Pneumologia, v. 33, p. 572-578, 2007. 
PRADO, T. N. et al. Prevalence and risk factors for latent tuberculosis infection among primary health care workers in Brazil. Cadernos de saúde pública, v. 33, p. e00154916, 2017.

SAUNDERS, M. J. et al. A score to predict and stratify risk of tuberculosis in adult contacts of tuberculosis index cases: a prospective derivation and external validation cohort study. The Lancet Infectious Diseases, v. 17, n. 11, p. 1190-1199, 2017.

SILVA, A. M. M. et al. Perfil epidemiológico da tuberculose na população indígena. Revista Brasileira de Educação e Saúde, v. 8, p.67 -71, 2018 a.

SILVA, D. R. et al. Risk factors for tuberculosis: diabetes, smoking, alcohol use, and the use of other drugs. Jornal Brasileiro de Pneumologia, v. 44, p. 145-152, 2018 b.

SILVA, E. R. A. et al. Fatores de risco para a tuberculose na população brasileira. Revista Coopex, v.10, p. 1 -10, 2019.

SILVA, L. M. S. et al. Controle da tuberculose no sistema prisional. Revista Coopex, v.7, p. 1$9,2016$.

TAVARES, E. R.; SOUSA, M. N. A. Percepções de enfermeiros sobre a importância do controle e tratamento da tuberculose no sistema prisional. Revista Ciência \& Desenvolvimento, v. 7, n. 1, p.211-221, 2014.

TEMOTEO, R. C. A. et al. Recommendations and effectiveness of chemoprophylaxis of latent infection by mycobacterium tuberculosis. Revista de Enfermagem UFPE on line, v. 9, p. 9983-9993, 2015.

VAZ, A.; REIFEGERSTE, C. P. Fatores de risco ao abandono do tratamento da tuberculose em Santa Catarina. Rev. méd. Paraná, v. 77, n. 2, p. 22-26, 2019.

YEN, Y. et al. Factors associated with delayed recognition of pulmonary tuberculosis in emergency departments in Taiwan. Heart \& Lung, v. 44, n. 4, p. 353-359, 2015.

ZENTENO-CUEVAS, R. et al. Co-infection and risk factors of tuberculosis in a Mexican HIV+ population. Revista da Sociedade Brasileira de Medicina Tropical, v. 44, p. 282-285, 2011.

Recebido em: 10/10/2021

Aprovado em: 05/11/2021

Publicado em: 12/11/2021 